\title{
Endovascular Treatment of Ophthalmic Artery Aneurysms: Assessing Balloon Test Occlusion and Preservation of Vision in Coil Embolization
}

\author{
J.H. Ahn, Y.D. Cho, H.-S. Kang, J.E. Kim, W.-S. Cho, S.C. Jung, C.H. Kim, and M.H. Han
}

\begin{abstract}
BACKGROUND AND PURPOSE: ICA-ophthalmic artery aneurysms have unique configurations corresponding to relative risks of ophthalmologic morbidities. Presented herein are clinical and radiologic outcomes of coil embolization in ophthalmic artery aneurysms.
\end{abstract}

MATERIALS AND METHODS: From January 2003 to September 2013, endovascular coiling was performed in 43 consecutive patients with ophthalmic artery aneurysms, each classified by the degree to which the ophthalmic artery was incorporated by the aneurysm and the contiguity between the ophthalmic artery entry and parent ICA. Clinical and radiologic outcomes of this approach were investigated, including the technical aspects of treatment and the efficacy of balloon test occlusion.

RESULTS: Among 14 patients (32.6\%, all ophthalmic artery types) undergoing balloon test occlusion before endovascular coiling, patent collaterals between the external carotid artery and ophthalmic artery were demonstrated in $12(85.7 \%)$ and complete compromise of the ophthalmic artery (without affecting vision) occurred in 4 patients during coiling. Steam-shaped S-configured (67.9\%) or straight microcatheters (17.8\%) facilitated aneurysm selection in most of the superiorly directed ophthalmic artery aneurysms $(n=28)$, and steamshaped pigtail microcatheters (85.7\%) were useful in medially directed aneurysms $(n=14)$. Balloon protection $(n=22)$ was generally used to facilitate coiling, or a stent $(n=9)$ was alternatively deployed. Satisfactory aneurysmal occlusion was achieved through coil embolization in 37 lesions (86.1\%). During follow-up of 35 patients (mean interval, $12.9 \pm 9.4$ months), only 1 instance $(2.9 \%)$ of major recanalization was observed.

CONCLUSIONS: If one tailors technical strategies, ophthalmic artery aneurysms are amenable to safe and effective endovascular coil embolization, which tends to be stable in follow-up. Balloon test occlusion may be helpful in devising treatment strategies to preserve vision when coiling ophthalmic artery aneurysms (especially those incorporating an ophthalmic artery origin) is done.

ABBREVIATIONS: $\mathrm{BTO}=$ balloon test occlusion; $\mathrm{ECA}=$ external carotid artery; $\mathrm{OA}=$ ophthalmic artery

D espite considerable progress in microsurgical techniques, surgical treatment of ICA-ophthalmic artery aneurysms (OAs) is challenging, given their proximity to the optic apparatus, anterior clinoid process, and cavernous sinus. The recently devel-

Received January 17, 2014; accepted after revision March 31.

From the Department of Neurosurgery (J.H.A.), Hallym University Sacred Heart Hospital, Hallym University College of Medicine, Anyang, Korea; Departments of Radiology (Y.D.C., M.H.H.) and Neurosurgery (H.-S.K., J.E.K., W.-S.C., M.H.H.), Seoul National University Hospital, Seoul National University College of Medicine, Seoul, Korea; Department of Radiology (S.C.J.), Asan Medical Center, Ulsan University College of Medicine, Seoul, Korea; and Department of Neurology (C.H.K.), Stroke Center, Myongji Hospital, Goyang, Korea.

This study was supported by a grant from the Korea Healthcare Technology R\&D Project, Ministry for Health, Welfare and Family Affairs, Republic of Korea (A111101). Please address correspondence to Young Dae Cho, MD, Department of Radiology, Seoul National University Hospital, Seoul National University College of Medicine, 101 Daehak-ro, Jongno-gu, Seoul 110-744, Korea; e-mail: aronnn@naver.com

- Indicates open access to non-subscribers at www.ajnr.org

http://dx.doi.org/10.3174/ajnr.A3999 oped neurointerventional arm enables an endovascular approach in this setting as an alternative to surgical clipping and the relatively high morbidity it entails. Nevertheless, the risk of impaired vision due to unexpected thromboembolism or occlusion of the OA after coiling is still a major concern. ${ }^{1-3}$ Earlier investigations of endovascular treatment generally have considered OA lesions a subset of paraclinoid aneurysms. ${ }^{4-7}$ Furthermore, such reviews have not fully addressed vision as an outcome, including angiographic assessment of collateral circulation before treatment. In this study, only aneurysms originating at the orifice of the OA are presented; we focused on angiographic and clinical aspects of vision as a measure of therapeutic outcome.

\section{MATERIALS AND METHODS Study Population}

Between January 2003 and September 2013, a total of 2654 patients with 3163 intracranial aneurysms were treated by endovascular methods at our institution. Among them, we identified 43 
patients with 43 OA aneurysms from the prospectively recorded data base. Paraclinoid ICA aneurysms apart from an OA origin and pure OA aneurysms without continuity with the ICA were excluded. All lesions were further classified by the degree to which the OA was incorporated by the aneurysm and the contiguity between the OA entry and the parent ICA. Aneurysms incorporating an OA origin were designated "OA type," whereas those adjacent to the OA but originating from the ICA were termed "ICA type" (Fig 1). Arterial branches associated with these aneu-

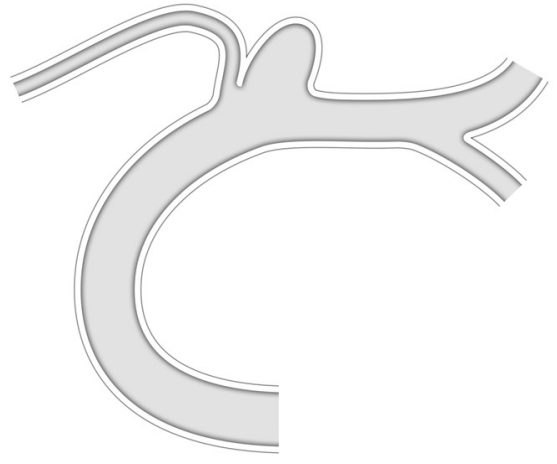

\section{A}

ICA type

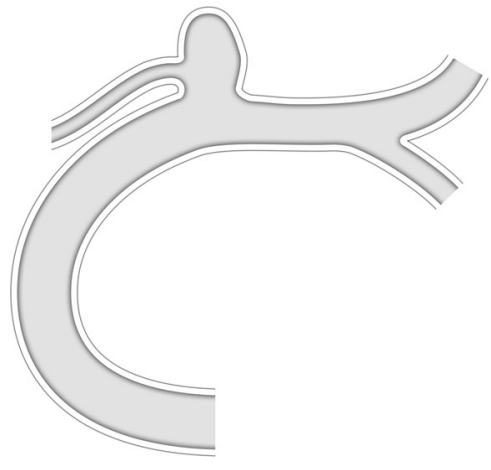

B
OA type
FIG 1. Classification of OA aneurysms (ICA type versus OA type).
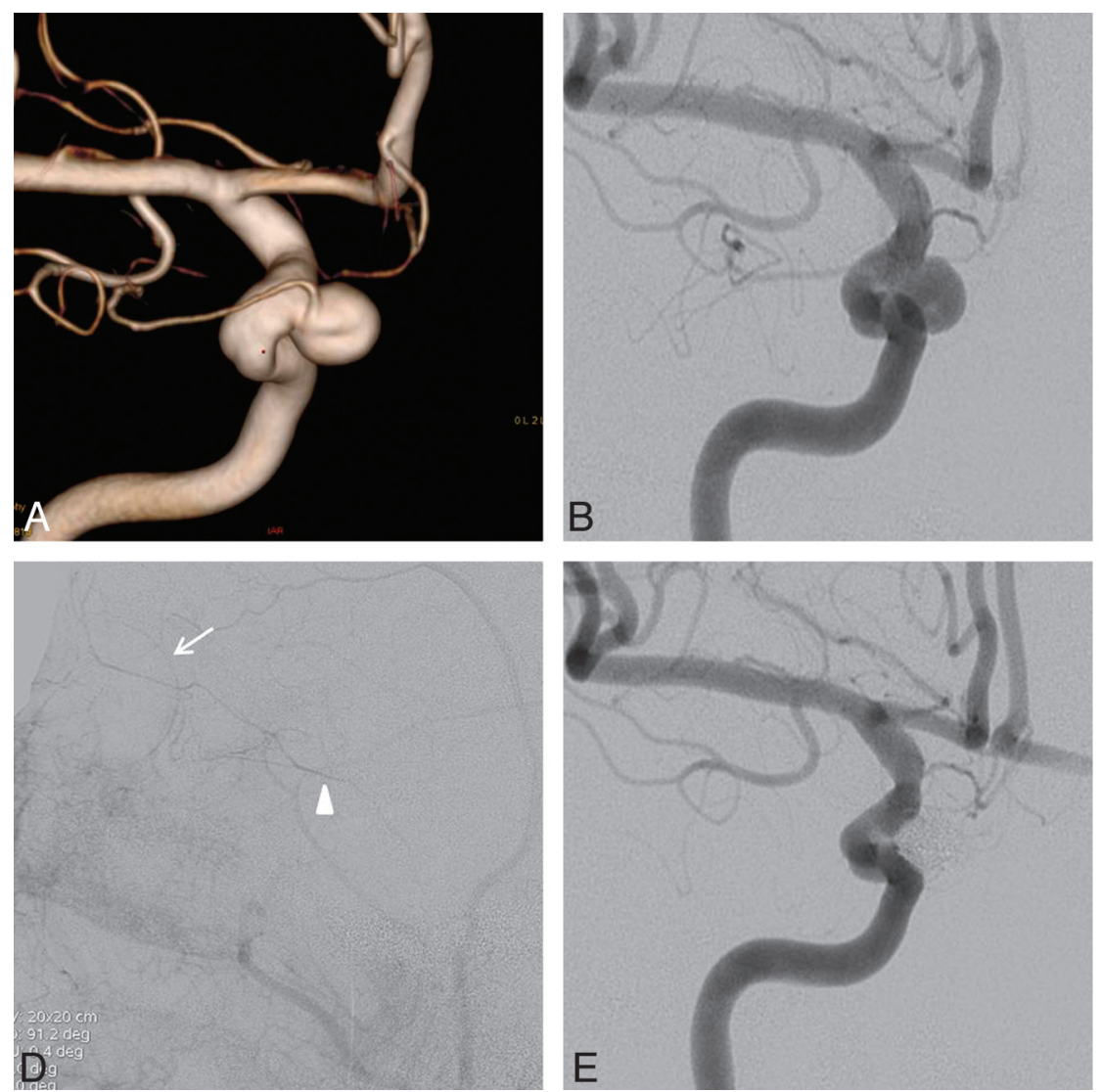

rysms were confirmed by digital subtraction angiography and 3D imaging. Therapeutic decisions were reached through multidisciplinary deliberation of both neurosurgeons and nonsurgical neurointerventionalists, and informed consent was obtained from all patients. The study protocol was approved by our institutional review board.

\section{Endovascular Procedures}

Most of the endovascular procedures were performed with the patient under general anesthesia; the remaining 8 patients had local anesthesia, which was limited to the patients with favorable arterial architecture and aneurysmal configuration for coil embolization and good cooperation. All patients underwent cerebral angiography and rotational angiography with $3 \mathrm{D}$ image reconstruction with Integris $\mathrm{V}$ (Philips Healthcare, Best, the Netherlands) or Innova IGS 630 scanners (GE Healthcare, Milwaukee, Wisconsin) to evaluate aneurysmal configuration and arterial vasculature. All patients were managed with an antiplatelet agent before coiling

FIG 2. $A$ and $B$, Conventional angiography and $3 D$ imaging of a medially directed $O A$ aneurysm (OA originating from the aneurysmal neck and an aneurysm incorporating most of the OA entry). C, Balloon test occlusion performed after planned sacrifice of the OA orifice during coil embolization; the balloon catheter was placed at the ophthalmic segment (arrow) and inflated. D, With the balloon inflated, angiographic image of the common carotid artery reveals choroidal blush (arrow) and contrast filling (arrowhead) of the OA through the middle meningeal artery. $E$, Final angiographic view of the occluded aneurysm and OA orifice. F, Angiography of the external carotid artery confirms a patent choroidal blush (arrow) and contrast filling of the OA (arrowhead) after coiling. 

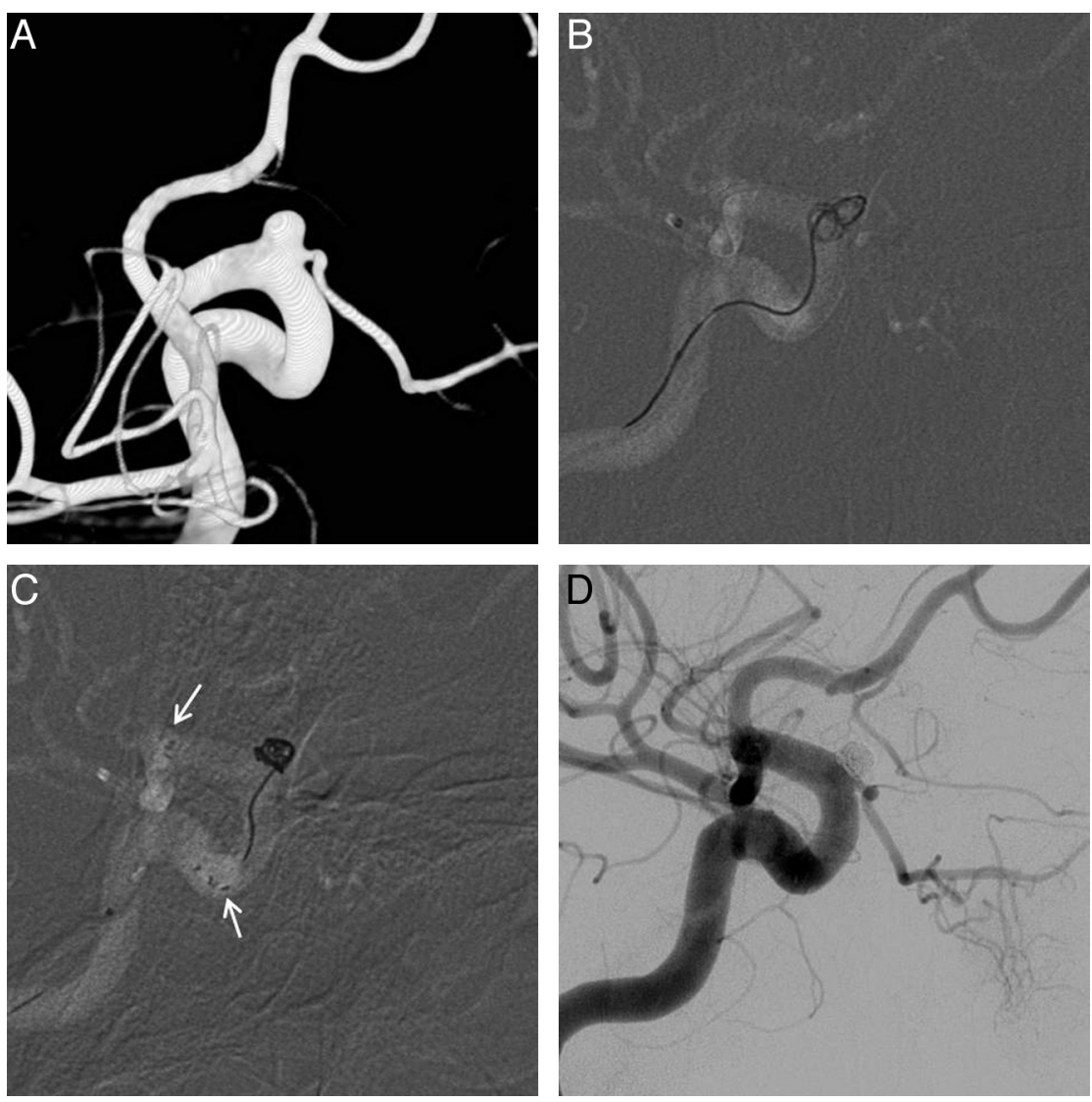

FIG 3. $A, 3 D$ imaging of a superiorly directed $O A$ aneurysm; balloon test occlusion was not performed due to the aneurysm type (ICA) and a relatively well-demarcated margin between the $\mathrm{OA}$ orifice and the aneurysmal neck in the working projection. $B$, Simple technique via a steamshaped S-configured microcatheter results in persistent herniation of coils into the parent artery. $C$, A Neuroform stent (arrows, stent markers) stabilizes the coil mass and secures the OA orifice. $D$, Completion angiography of successfully occluded aneurysm, with the OA preserved.

in a relatively consistent scheme at our institution. ${ }^{8}$ In all patients with unruptured aneurysms, a 300-mg loading dose of clopidogrel was given the day before the procedure, and an additional $75 \mathrm{mg}$ of clopidogrel was given the morning of the procedure. In patients with poor response to clopidogrel, based on the VerifyNow P2Y12 assay (Accumetrics, San Diego, California) (ie, P2Y12 reactivity unit of $>285$ ), a 300-mg loading dose of aspirin was added. Dual-agent antiplatelet therapy was administered if stent protection was anticipated. In patients showing poor response to clopidogrel, cilostazol (200 mg) was added. After femoral sheath placement, systemic heparinization was started with a loading dose of $3000 \mathrm{IU}$ and was maintained with a dose of 1000 IU hourly. In a patient with an acutely ruptured aneurysm, antiplatelet medication was not administered before the procedure. In these cases, systemic heparinization was initiated shortly after adequate protection of the aneurysm. After the procedure, antiplatelet medications were not routinely prescribed for maintenance except in case of stent protection, coil protrusion, and procedural thromboembolism.

\section{Assessment of Balloon Test Occlusion}

Baseline neurologic examinations were performed, with visual acuity assessed before and after each coiling procedure. After detailed analysis of the aneurysmal configuration and arterial course, the most appropriate therapeutic strategies were planned. Balloon test occlusion (BTO) was performed selectively and before endovascular treatment for the following reasons: 1) anticipated compromise of the OA entry during coiling, or 2) compromise of the OA unavoidable during coil embolization (ie, aneurysms incorporating much of the OA orifice or the OA originating from the aneurysmal dome) (Fig 2). If OA preservation was likely, based on aneurysm/OA properties favorable for endovascular coiling, BTO was not performed (Fig 3). The treatment algorithm for this cohort is depicted in a schematic (Fig 4).

BTO involved the following steps: 1) a guiding catheter placed in the common carotid artery; 2) a balloon catheter, HyperGlide (Covidien, Irvine, California and Scepter C (MicroVention, Tustin, California) $(4 \times 15 \mathrm{~mm}$ or $20 \mathrm{~mm}$ ), placed at the ophthalmic segment of the ICA, including OA origin; 3 ) careful inflation of a balloon with salinediluted contrast media under fluoroscopic observation; and 4) contrast instilled (on ICA occlusion) through the common carotid artery to evaluate collateral flow between the external carotid artery (ECA) and the OA. A positive result with BTO was defined as retrograde filling of the OA main trunk via ECA branches and demonstration of retinochoroidal blush (Fig 2). In BTO-positive patients, occlusion of the aneurysm and compromise of the OA orifice were attempted in combination. Once coiling was complete, collateral flow was re-affirmed by ECA angiography. With negative BTO results, we elected one of the following options on the basis of aneurysmal configuration and size: 1) coil embolization by using various protective devices to preserve OA flow (intentional partial protective embolization on occasion), 2) no treatment and follow-up, or 3) surgical clipping.

\section{Angiographic Outcome and Follow-Up}

Immediate angiographic results after coil embolization were classified according to a 3-point Raymond scale: complete obliteration (no residual filling of contrast medium in the aneurysms), residual neck (the persistence of any portion of the original defect of the arterial wall), and residual aneurysm (any opacification of the sac). ${ }^{9}$

Clinical outcomes were evaluated with the Glasgow Outcome Scale, which was applied throughout hospitalization and at the last available clinical follow-up. In patients with unruptured aneurysms, MR angiography with 3D reconstruction was recommended 6, 12, 24, and 36 months after coil embolization. Additionally, plain radiography was recommended at 1 and 3 months 


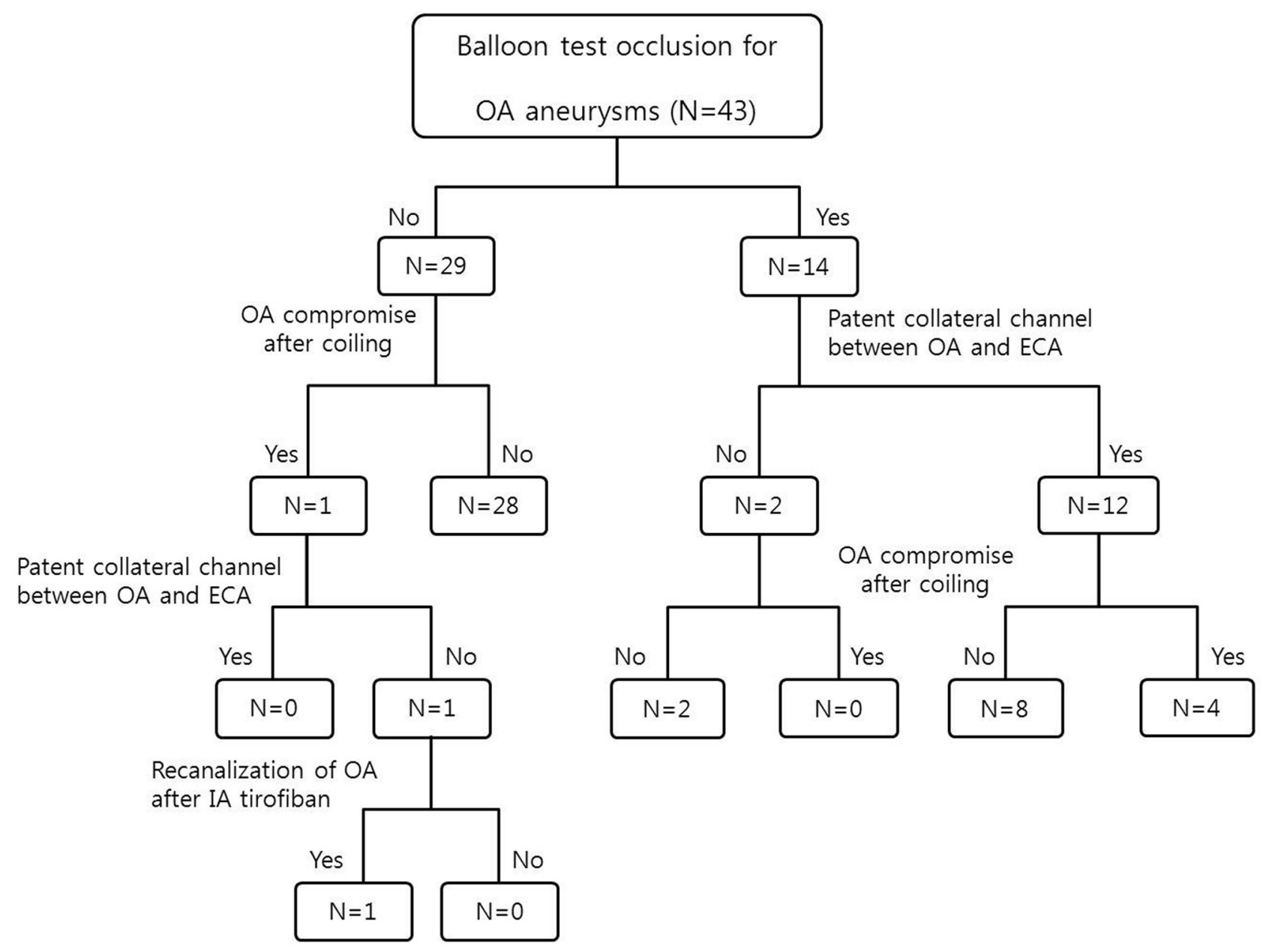

FIG 4. Schematic depicting the treatment algorithm in the study population.

postembolization in patients with ruptured aneurysms. Two experienced neurointerventionalists (M.H.H., H.-S.K.) who were blinded to the immediate postembolization angiogram independently reviewed anatomic follow-up results and categorized them as follows: stable occlusion (no interval change since the procedure or progressive thrombosis within the aneurysm), minor recanalization (progressive filling limited to the neck of the aneurysm), and major recanalization (aneurysmal sac filling). Conventional angiography and repeat embolization were recommended for patients showing major recanalization.

\section{RESULTS}

\section{Population and Aneurysmal Characteristics}

General characteristics of the study population $(n=43)$ undergoing endovascular coil embolization for OA aneurysms are summarized in Table 1. The mean patient age at the time of the procedure was $53.3 \pm 10.4$ years (range, $30-74$ years), with a male/ female ratio of 1:3.3. Nearly all OA aneurysms (97.6\%) were discovered incidentally, with 1 exception in which the patient presented with subarachnoid hemorrhage.

Maximal diameters of aneurysms treated ranged from 2.7 to $15.7 \mathrm{~mm}$ (mean, $5.6 \pm 2.7 \mathrm{~mm}$ ), and $53.5 \%$ of all lesions were $<5$ $\mathrm{mm}$. There were 19 aneurysms of the ICA type and 24 of the OA type. Additional aneurysms existed in 18 patients (41.9\%). Most lesions were wide-neck $(38 / 43,88.4 \%)$, with depth-to-neck ratios
$<1.5$. The aneurysms occupied either the superior wall of the ICA $(28 / 43,65.1 \%)$, the medial wall $(14 / 43,32.6 \%)$, or the inferior wall $(1 / 43,2.3 \%)$.

\section{Technical Aspects of Endovascular Treatment and Anatomic Outcomes}

Treatment techniques applied to this series of OA aneurysms are shown in Table 2. The microcatheters used to select the aneurysms differed, depending on the aneurysm location and direction and parent artery course. In aneurysms of the superior wall, steam-shaped S-configured microcatheters (19/28, 67.9\%) (Fig 3) or straight microcatheters $(5 / 28,17.9 \%)$ were used in most patients. In lesions of the medial wall, primarily steam-shaped pigtail microcatheters $(12 / 14,85.7 \%)$ were used (Fig 2).

Regarding other technical aspects, balloon remodeling was most frequently used $(22 / 43,51.3 \%)$, followed by stent protection $(9 / 43,20.9 \%)$ and either a multiple-microcatheter or single-microcatheter technique $(6 / 43,13.9 \%$, each). All implanted stents were Neuroform devices (Stryker Neurovascular, Fremont, California) with open cells (Fig 3). Immediate angiographic assessment revealed occlusion in 14 aneurysms (32.6\%), residual neck in $23(53.5 \%)$, and residual aneurysm in 6 (13.9\%).

Although an asymptomatic thrombus did occur in 1 patient, no other procedure-related adverse events were encountered. All 
Table 1: OA aneurysms: general characteristics and outcomes of endovascular coiling

\section{Characteristics}

\begin{tabular}{lc}
\hline No. of aneurysms and patients & 43 aneurysms, 43 patients \\
Age (yr) (mean) & $53.3 \pm 10.4$ \\
Female/male & $33: 10$ \\
Presentation & \\
Incidental & 42 \\
Visual disturbance & 0 \\
Ruptured & 1 \\
Aneurysm direction & \\
Superior & $28(65.1 \%)$ \\
Medial & $14(32.6 \%)$ \\
Inferior & $1(2.3 \%)$ \\
Aneurysm type & \\
ICA type & $19(44.2 \%)$ \\
OA type & $24(55.8 \%)$ \\
Aneurysm size & \\
$\leq 5$ mm & $23(53.5 \%)$ \\
$5 \sim 10$ mm & $18(41.8 \%)$ \\
$\geq 10$ mm & $2(4.7 \%)$ \\
No. of aneurysms of other locations & \\
OA aneurysm only & $25(58.1 \%)$ \\
Multiple aneurysm & $18(41.9 \%)$ \\
Depth-to-neck ratio & \\
$\leq 1$ & $22(51.2 \%)$ \\
$1.0 \sim 1.5$ & $16(37.2 \%)$ \\
$\geq 1.5$ & $5(11.6 \%)$ \\
Direction of aneurysm & \\
Superior & $27(62.8 \%)$ \\
Medial & $14(32.5)$ \\
Other & $2(4.7 \%)$ \\
Initial occlusion result & \\
Complete occlusion & $14(32.6 \%)$ \\
Residual neck & $23(53.5 \%)$ \\
Residual aneurysm & $6(13.9 \%)$ \\
Sollow-up result & \\
Minor recanalization & $30(85.7 \%)$ \\
Major recanalization & $4(11.4 \%)$ \\
\hline & $1(2.9 \%)$ \\
\hline
\end{tabular}

Table 2: Technical strategies and directional shaping of

\begin{tabular}{lccc} 
microcatheters & $\begin{array}{c}\text { Superior } \\
(\boldsymbol{n}=\mathbf{2 8})\end{array}$ & $\begin{array}{c}\text { Medial } \\
(\boldsymbol{n}=\mathbf{1 4})\end{array}$ & $\begin{array}{c}\text { Inferior } \\
(\boldsymbol{n}=\mathbf{1})\end{array}$ \\
\hline Technique & & & \\
$\quad$ Single microcatheter & 5 & 1 & 0 \\
$\quad$ Multiple microcatheter & 4 & 2 & 0 \\
Balloon remodeling & 15 & 7 & 0 \\
$\quad$ Stent protection & 4 & 4 & 1 \\
Microcatheter shape & 19 & 2 & 0 \\
$\quad$ Steam-shaped S & 5 & 0 & 0 \\
Straight & 3 & 10 & 1 \\
$\quad$ Steam-shaped pigtail & 1 & 2 & 0 \\
$\quad$ Preshaped $45^{\circ}$ or $90^{\circ}$ & & &
\end{tabular}

patients were neurologically intact (Glasgow Outcome Scale score 5) at the time of discharge.

\section{Balloon Test Occlusion and Clinical Outcomes}

In approximately one-third (14/43, 32.6\%) of the cohort (all OA type aneurysms, representing 58.3\% [14/24] of patients with OA type aneurysms), BTO was performed without complications before the coiling procedure, confirming patent collateral channels between the ECA and OA in 12 patients (85.7\%). Complete com- promise of the OA (without a decline in vision) occurred in 4 of these 12 patients (Fig 2) because of endovascular coiling. The other 8 patients with favorable collaterals by BTO showed persistent antegrade filling of the OA with contrast and choroidal blush, despite protrusion of the coil into the OA entry. All patients received antiplatelet medication for at least 1 month.

Among the 29 patients not undergoing BTO, unexpected compromise of flow to the OA occurred in 1 patient during endovascular coiling. ECA angiography was performed immediately, and there was no filling of the OA through the ECA. Intraarterial tirofiban $(0.75 \mathrm{mg})$ then was infused to resolve the thrombus, and flow was restored (Fig 5). After the coiling procedure, an intravenous infusion of tirofiban was maintained for 12 hours and antiplatelet medication was administered for 1 month. The patient had no symptoms related to vision.

\section{Follow-Up Results}

Follow-up radiologic imaging at least 6 months after coil embolization was performed in 35 aneurysms (mean interval, $12.9 \pm 9.4$ months), with the exception of 3 recently treated aneurysms $(<6$ months). Thus, the follow-up rate was $87.5 \%$ (35/40). On the basis of follow-up imaging, stable occlusion was observed in 30 aneurysms (87.5\%); minor recanalization, in 4 (11.4\%); and major recanalization, in 1 (2.9\%). One patient with major recanalization underwent repeat endovascular coiling. Follow-up angiography performed 12 months after re-embolization showed stable occlusion. During the follow-up period, none of the patients had delayed cerebral infarction.

\section{DISCUSSION}

A number of terms are used to describe aneurysms of the ICA segment between the $\mathrm{OA}$ and posterior communicating artery, namely paraophthalmic, paraclinoid, and ophthalmic segment aneurysms. Such lesions represent approximately $1.5 \%-8.0 \%$ of all intracranial aneurysms ${ }^{5,10,11}$ and show a marked female preponderance (in most series) and a strong association with multiple aneurysms. ${ }^{5,6,11}$ These demographics, including a propensity for multiple lesions, were shared by our patients, all of whom had aneurysms originating at the orifice of the OA. The tendency to rupture was also relatively low. Only 1 of our subjects presented with subarachnoid hemorrhage.

BTO of the ICA has become an established means of evaluating the adequacy of collateral flow in patients with large/giant aneurysms or head and neck tumors involving the carotid artery, in which arterial sacrifice or prolonged transient occlusion is expected during surgical or endovascular therapy. ${ }^{12}$ However, research aimed at estimating the adequacy of collateral flow from the ECA to the OA or correlating vision outcomes with pretreatment angiographic studies of collateral circulation to the $\mathrm{OA}$ is scarce. Shaibani et $\mathrm{al}^{13}$ performed BTO in 2 patients, each with an aneurysm incorporating the OA into its neck. They gauged the adequacy of collateral flow by deterioration of vision, rather than by confirming collateral circulation as we did through angiography. Both patients exhibited deteriorating vision within 6 minutes after balloon inflation that resolved on deflation, thus signifying insufficient collateral blood flow and an indication for surgical clipping. However, this method carries some potential limitations 

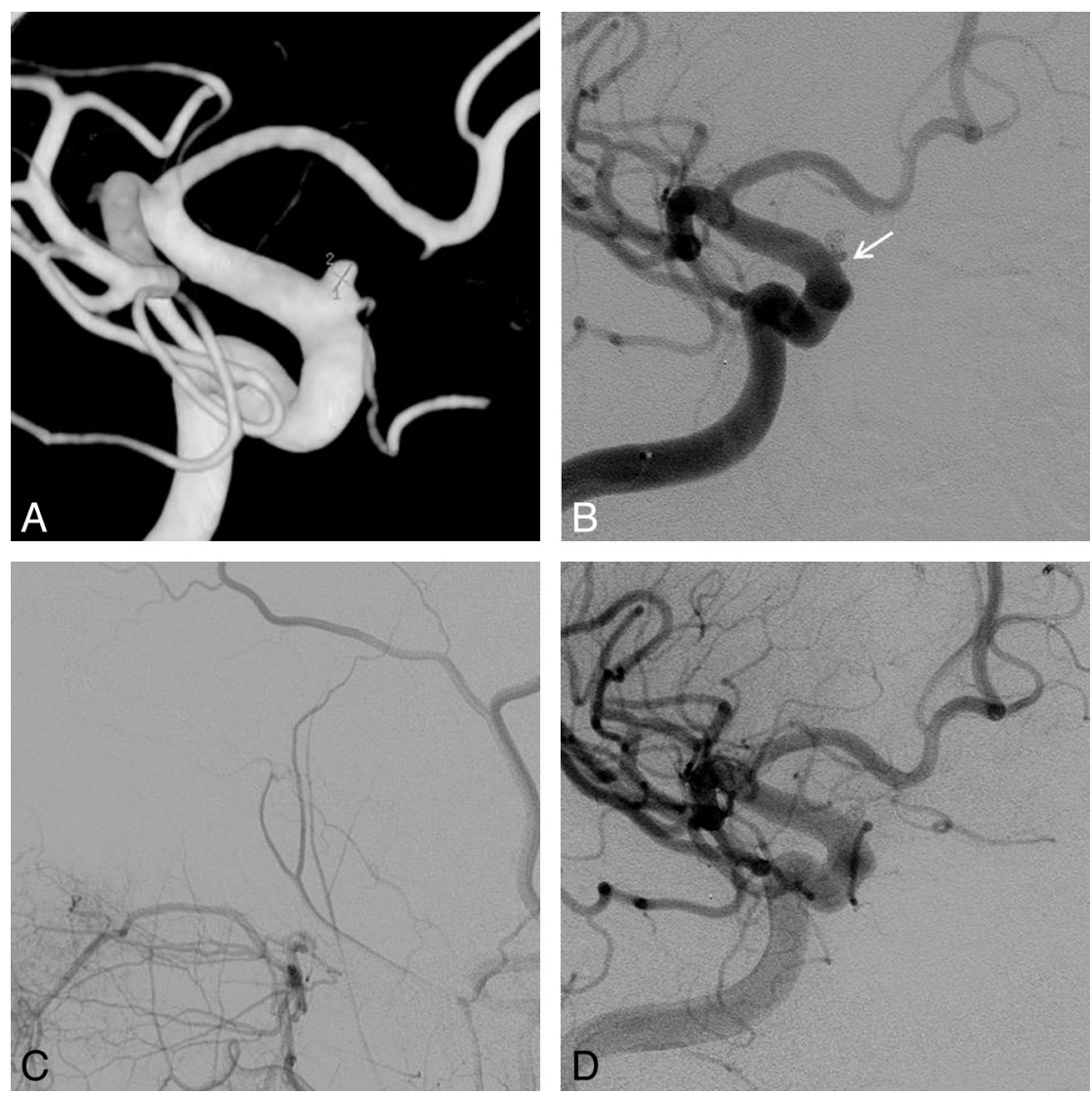

FIG 5. A, $3 D$ imaging of a superiorly directed $O A$ aneurysm; balloon test occlusion was not performed due to the aneurysm type (ICA) and relatively well-demarcated margin, $B$, Completion angiography of a successfully occluded aneurysm with OA compromise (arrow). C, Angiography of the external carotid artery confirms no patent contrast filling of the OA. D, Restoration of OA flow after intra-arterial tirofiban infusion $(0.75 \mathrm{mg})$.

and risks, despite its apparent utility. The authors were concerned that perhaps duration of BTO was critical for accurate assessment. In other words, collateral flow from the ECA may have been evident with longer occlusion. Mathis et $\mathrm{al}^{12}$ similarly performed continuous neurologic monitoring of 500 patients throughout 15 -minute periods of test occlusion. Here, 16 patients $(3.2 \%)$ had complications during BTO, including dissection, thromboembolism, and pseudoaneurysm.

No patients had complications of BTO in our hands, compared with the above study, which may attest to the merits of highly compliant balloons, less procedural time, and a simple procedure for confirming collateral flow from the ECA to the OA. Likewise, none of our patients experienced visual complications after complete OA compromise, gauged by our angiographic criteria (ie, OA flow determination and retinochoroidal blush by ECA-OA collaterals). Our study illustrates that the use of BTO to confirm collateral flow is seemingly innocuous and may provide a reliable index of postprocedural vision integrity, despite the limited sampling of patients involved. Because OA filling via the ECA does not precisely reflect the perfusion status of the central retinal artery and retinochoroidal blush also stems from the posterior ciliary artery, both criteria are important guides. None of our BTO-negative patients had OA compromise, so outcomes in terms of vision were not compared by positive and negative BTObased subsets. Still, this study does show that vision is preserved after OA compromise in BTO-positive patients. Concomitant monitoring with electroretinography or visual evoked potentials may also be helpful in predicting retinal ischemia or delayed thromboembolic events.

Collateral channels between the ECA and $\mathrm{OA}$ prevent permanent blindness after occlusion of the OA in $90 \%$ of patients. ${ }^{14,15}$ In our study, the rate of patent collateral channels between the $\mathrm{OA}$ and ECA $(84.6 \%)$, confirmed by BTO, was similar to that in previous reports. However, thrombotic occlusion at the OA orifice is a major concern during the coil embolization procedure itself and in the follow-up period. Should a thrombus unexpectedly form during coil embolization in patients who have not been evaluated by BTO, ECA angiography should be performed immediately in search of collateral channels between the ECA and OA. Although the probability of patent collaterals is high, the potential risk of ocular ischemia after coiling cannot be ignored. In this study, 1 instance of unexpected thrombus did occur in which there was no demonstrable filling of the OA through the ECA. Intra-arterial tirofiban infusion subsequently restored flow to the OA, but ocular ischemia may result from thromboembolism and not from loss of OA perfusion alone. Ischemia from hypoperfusion ostensibly is avoidable by confirming collateral flow through BTO. In 8 patients with positive BTO results, initially slated for OA sacrifice, antegrade flow through the OA persisted, despite intentional OA compromise. We worried that incomplete compromise of the OA, with coil protrusion into the OA entry, might be a source of thromboembolism. Therefore, these patients were advised to continue anticoagulation and antiplatelet medication after the procedures.

Unfavorable aneurysmal configurations, including wide-neck lesions and those in which OA entry is incorporated into the aneurysmal neck, constitute a persistent challenge for endovascular treatment. Coil embolization with OA preservation is especially difficult in OA type aneurysms. The shape of the microcatheter used to select the aneurysm depends on aneurysm direction and the parent artery course. OA aneurysms are uniformly situated at the distal carotid siphon with sharp curves, so the direction of the microcatheter is inescapably affected by the curve of the carotid siphon. In superiorly directed aneurysms, steamed-shaped S-configured and straight microcatheters were generally used, whereas straight microcatheters were particularly helpful in aneurysms more proximally situated at the carotid siphon. In aneurysms directed medially, steam-shaped pigtail microcatheters were beneficial. Steamed-S and steamed-pigtail microcatheters tend to offer less resistance to kick-back during coil insertion. Thus, balloon protection or stent assistance was often required, not only for 
neck protection but also for bolstering the microcatheter. In all instances in which stents were deployed, Neuroform devices were used exclusively. We wanted the struts of this open-cell stent to herniate into the aneurysmal neck and preserve the OA entry (Fig 3).

Relative to other locations, occlusion immediately following coil embolization seemed more difficult to achieve in the OA aneurysms of this series, possibly due to incorporation of the OA into these aneurysms and their proximity to the carotid siphon. In any event, anatomic outcomes were excellent at follow-up, possibly influenced by aneurysmal configurations (ie, sidewall aneurysms) and hemodynamic buffering by the carotid siphon.

\section{CONCLUSIONS}

Because of their unique configurations and related ophthalmologic morbidities, OA aneurysms are considered a challenge for endovascular coiling. Balloon test occlusion seems helpful in planning treatment strategies for coiling of OA aneurysms that preserve vision. Through tailored techniques for shaping and insertion of microcatheters, excellent clinical and radiologic outcomes in OA aneurysms are possible by this method.

\section{ACKNOWLEDGMENTS}

We thank Yoon-Kyung Choi for preparation of the illustrations.

Disclosures: Moon Hee Han—RELATED: Consultancy: MicroVention Inc.

\section{REFERENCES}

1. Castillo B Jr, De Alba F, Thornton J, et al. Retinal artery occlusion following coil embolization of carotid-ophthalmic aneurysms. Arch Ophthalmol 2000;118:851-52

2. Ferrell AS, Lessne ML, Alexander MJ, et al. Visual complications after stent-assisted endovascular embolization of paraophthalmic and suprasellar variant superior hypophyseal aneurysms: the Duke
Cerebrovascular Center experience in 57 patients. World Neurosurg 2012;78:289-94

3. Sherif C, Gruber A, Dorfer C, et al. Ruptured carotid artery aneurysms of the ophthalmic (C6) segment: clinical and angiographic long term follow-up of a multidisciplinary management strategy. J Neurol Neurosurg Psychiatry 2009;80:1261-67

4. Heran NS, Song JK, Kupersmith MJ, et al. Large ophthalmic segment aneurysms with anterior optic pathway compression: assessment of anatomical and visual outcomes after endosaccular coil therapy. J Neurosurg 2007;106:968-75

5. Roy D, Raymond J, Bouthillier A, et al. Endovascular treatment of ophthalmic segment aneurysms with Guglielmi detachable coils. AJNR Am J Neuroradiol 1997;18:1207-15

6. Sengupta RP, Gryspeerdt GL, Hankinson J. Carotid-ophthalmic aneurysms. J Neurol Neurosurg Psychiatry 1976; 39:837-53

7. Yadla S, Campbell PG, Grobelny B, et al. Open and endovascular treatment of unruptured carotid-ophthalmic aneurysms: clinical and radiographic outcomes. Neurosurgery 2011;68:1434-43

8. Cho YD, Lee WJ, Kim KM, et al. Endovascular coil embolization of middle cerebral artery aneurysms of the proximal (M1) segment. Neuroradiology 2013;55:1097-102

9. Roy D, Milot G, Raymond J. Endovascular treatment of unruptured aneurysms. Stroke 2001;32:1998-2004

10. Almeida GM, Shibata MK, Bianco E. Carotid-ophthalmic aneurysms. Surg Neurol 1976;5:41-45

11. Drake CG, Vanderlinden RG, Amacher AL. Carotid-ophthalmic aneurysms. J Neurosurg 1968;29:24-31

12. Mathis JM, Barr JD, Jungreis CA, et al. Temporary balloon test occlusion of the internal carotid artery: experience in $\mathbf{5 0 0}$ cases. AJNR Am J Neuroradiol 1995;16:749-54

13. Shaibani A, Khawar S, Bendok B, et al. Temporary balloon occlusion to test adequacy of collateral flow to the retina and tolerance for endovascular aneurysmal coiling. AJNR Am J Neuroradiol 2004;25: 1384-86

14. Johnson HC, Walker AE. The angiographic diagnosis of spontaneous thrombosis of the internal and common carotid arteries. $\mathrm{J} \mathrm{Neu}$ rosurg 1951;8:631-59

15. Dehdashti AR, Safran AB, Martin JB, et al. Intraorbital ophthalmic artery aneurysm associated with basilar tip saccular aneurysm. Neuroradiology 2002;44:600-03 